\title{
Enumeration Class of Polyominoes Defined by Two Column
}

\author{
Eman f. Mohommed ${ }^{1}$, Haslinda Ibrahim ${ }^{2}$, Nazihah Ahmad $^{3}$ \\ ${ }^{I}$ School of Quantitative Sciences, College of Arts and Sciences, Universiti Utara Malaysia 06010 Sintok, \\ Kedah, Malaysia. \\ ${ }^{2}$ School of Quantitative Sciences, College of Arts and Sciences, Universiti Utara Malaysia 06010 Sintok, \\ Kedah, Malaysia. \\ ${ }^{3}$ School of Quantitative Sciences, College of Arts and Sciences, Universiti Utara Malaysia 06010 Sintok, \\ Kedah, Malaysia.
}

\begin{abstract}
Abacus diagram is a graphical representation for any partition $\mu$ of a positive integer $t$. This study presents the bead positions as a unite square in the graph and de ne a special type of e-abacus called nested chain abacus $\mathfrak{N}$ which is represented by the connected partition. Furthermore, we redefined the polyominoes as a special type of e-abacus diagram. Also, this study reveals new method of enumerating polyominoes special design when $e=2$.
\end{abstract}

Mathematics Subject Classification: 05A15

Keywords: Abacus, Polyominoes, n-connected square, enumeration, Free enumeration, Combinatorial.

\section{Introduction}

The enumeration of polyominoes is one of the studied subject in combinatorics. In 1953, Soloman Golomb introduced the term polyomino, and it was popularized by Gandner in 1957. Polyominoes is defined as a finite collection of $n$-monomino in which the monomino is a unite square in the plan $\mathrm{Z} \times \mathrm{Z}$. The general enumeration problem of polyominoes has not been solved yet, and it remains open to solution. There are three common ways of distinguishing polyominoes from $n$-monomino for enumeration which are, free polyominoes, one side and fixed polyominoes. Free polyominoes or $\mathrm{P}(n)$ are considered different distinct when none is a rotation or translation of another while their location or orientation is not important in the plan Fixed polyominoes are consider distinct when if they have different shape [9], [10], [14]. To the best of our knowledge, no one has attempted to find the enumeration of fixed polyominoes or $n$-connected square with 2 columns. Hence, this study proposes new method to find polyominoes by using nested chain abacus, and enumeration of n-connected square (polyominoes) which is a representation of connected bead positions sequences.

\section{Preliminary Notes}

In this section, we will redefine polyominoes as nested chain abacus. The first step is to examine the structure of abacus diagram, and, in the subsequent section, we discuss some important definitions. Nested chain abacus is a graphical representation of nonincreasing sequence $\mu^{(e, r)}=\left(\mu_{1}, \mu_{2}, \ldots, \mu_{\mathrm{b}}\right)$ called connected partition. To define the nested chain abacus $\mathfrak{N}$, we must first determine the value of $e$. where $e$ represents the number of the columns in the abacus which are labelled from 0 to $e-1$ and $e \geq 1$. The bead positions are located across the columns on the nested chain abacus which are labelled from left to right and continued to lower from top starting with 0 , the bead positions $n e, n e+1, n e+2,(n+1) e-1$ are located in row $n$ in the nested chain abacus. The process to define abacus is much easier if it involves usage of a graph. We can think of our abacus in the fourth quadrant of a standard two-dimension plane and any bead as a unit square in two-dimension plane. In following section, we define some important definitions foundation of this work. 
Definition 2.1. The bead positions $\beta_{i}$ is a sequence of four vertices with length 1, These vertices are connected by horizontal or vertical vertex edges with length 1 such that any two vertices are collinear. The vertices are a coor-dinates in the fourth quadrant of two-dimension plane, thus

$\beta_{i}=n \mathrm{e}+c=\{(c,-n),(c+1,-n),(c,-n-1),(c+1,-n-1) \mid 0 \leq \mathrm{c} \leq \mathrm{e}-$ 1 and $0 \leq \mathrm{n} \leq \boldsymbol{r}-\mathbf{1}\}$

where $1 \leqslant i \leqslant b$.

In this following section we will redefine polyominoes as nested chain abacus.

\subsection{Beta number sequence}

Let $\beta_{h}$ and $\beta_{k}$ are two beads. $\beta_{h}$ is bead represents in the fourth quadrant of standard two-dimension plan with four coordinates $(c,-n)(c+1,-n)$ $(c,-n-1)(c+1,-n-1)$, then $\beta_{k}$ would be a unit square in the fourth quadrant of standard two-dimension plan with one of the following coordinates:

1. If $(c+1,-n),(c+2,-n),(c+1,-n-1),(c+2,-n-1)$ then $\beta_{k}$ and $\beta_{h}$ are connected beads, see Figure 1.a.

2. If $(c,-n-1),(c,-n-2),(c+1,-n-1),(c+1,-n-2)$ then $\beta_{k}$ and $\beta_{h}$ are connected beads, see Figure 1.b.

3. If $(c,-n),(c,-n+1),(c+1,-n),(c+1,-n+1)$ where $n \leqslant-1$ then $\beta_{k}$ and $\beta_{h}$ are connected beads, see Figure 1.c.

4. If $(c-1,-n),(c,-n),(c-1,-n-1),(c,-n-1)$ where $c \geqslant 1$ then $\beta_{k}$ and $\beta_{h}$ are connected beads, see Figure 1.d.

5. If $(c+y,-n),(c+y+1,-n),(c+y,-n-1),(c+y+1,-n-1)$ where $y$ is a positive integer then $\beta_{k}$ and $\beta_{h}$ are disconnected beads, see Figure 1.e.

6. If $(c-y,-n),(c-y-1,-n),(c-y,-n-1),(c-y-1,-n-1)$ where $0 \leqslant c-y-1$ and $y$ is a positive integer then $\beta_{k}$ and $\beta_{h}$ are disconnected beads, see Figure 1.f.

7. If $(c,-n-y-1),(c,-n-y-2),(c+1,-n-y-1),(c+1,-n-y-2)$ where $y$ is a positive integer then $\beta_{k}$ and $\beta_{h}$ are disconnected beads, see Figure 1.z.

8. If $\left(c+x,-n+x^{\prime}\right),\left(c+x+1,-n+x^{\prime}\right),\left(c+x+1,-n+x^{\prime}\right)$, $\left(c+x+1,-n+x^{\prime}-1\right)$ where $x$ is a positive integer, $0 \leqslant c+x$ and $x^{\prime} \leqslant n$ then $\beta_{k}$ and $\beta_{h}$ are disconnected beads, see Figure 1.j.

9. If ( $\left.c \mp v,-n \mp v_{r}\right)\left(c \mp v+1,-n \mp v_{r}\right)(c \mp v,-n \mp v,-1)(c \mp v+$ $1,-n \mp v,-1)$ then $\beta_{k}$ and $\beta_{h}$ are disconnected beads. Where $c \geqslant v$ and $v^{\prime} \leqslant n, 0 \leqslant c+x$ and $x^{\prime} \leqslant n$, see Figure 1.j. 


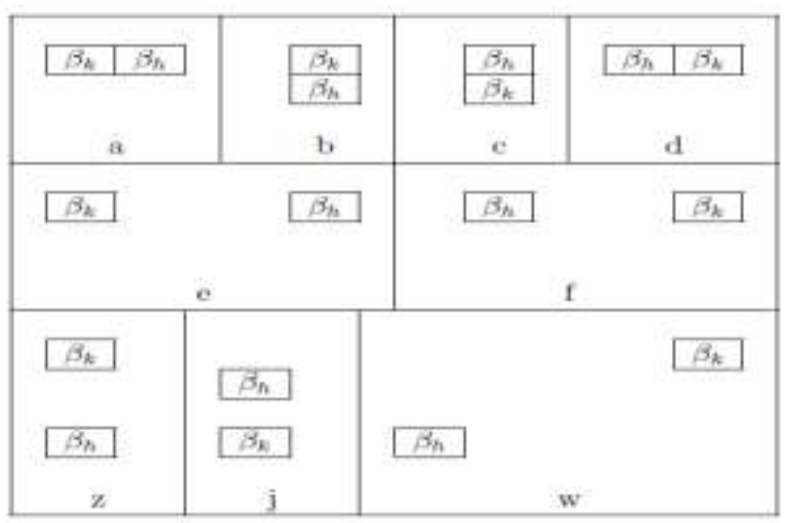

Figure 1: Connected and disconnected bet numbers

Definition 2.2. A path is a finite sequence of beads which are horizontal or vertical connected.

Definition 2.3. A sequence of beads are connected if there are path between each two of the beads in the nested chain abacus.

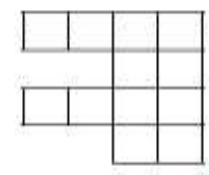

Figure 2: nested chain abacus where bead positions sequence is $\{0,1,2,3$ $, 6,7,8,9,10,11,14,15\}$ and $e=4$

Some combinatorial results after the redefinition of ployominoes as 2-abacus connected. In the next section, we use some combinatorial results to solve one of the ployominoes problems.

\section{Some combinatorial result}

No algorithm or rule or function is known, at the present time, to have been used for a number of existing polyominoes or $n$-connected square. Several algorithms are discovered to calculate the number of $n$-connected square which can be obtained if the number of $n$ is fixed integer number, but these algorithms become very tedious as $n$ increases. In this section, we intercede a class of nested chain abacus for $n$-connected square $\Im^{2}$ nested chain abacus, The class $\Im^{2}$ is defined as follows.

Definition 3.1. Let $\mathfrak{N}$ be the nested chain abacus of $n$-connected square with e columns. Then $\mathfrak{N}$ is called $\Im^{2}$ if $e=2$.

Example 3.2 and Example 3.3 illustrates the definition of $\Im^{2}$ nested chain abacus.

Example 3.2. Let $\mathfrak{N}$ be the nested chain abacus of 4-connected objects represented by the connected partition $\mu^{(2,3)}=\left(1,0^{3}\right)$ as shown in figure ??(a). Since $e=2$, then $\mathfrak{N}$ is of class $\Im^{2}$ as shown in Figure3(a).

Example 3.3. Let $\mathfrak{N}$ be the nested chain abacus of 4 -connected objects represented by the connected partition $\mu^{(3,2)}=\left(2^{2}, 0^{2}\right)$ as shown in figure??(b). Since $e=3$, then $\mathfrak{N}$ is not of class $\Im^{2}$ as shown in Figure3(b). 

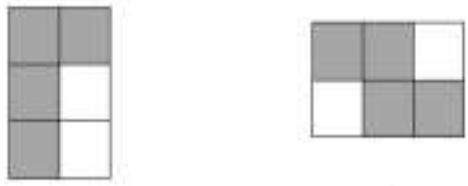

Figure 3: (a) 9 of 4-connected objects which is of class $3^{2}$ (b) 92 of 4-connected objocts which is not of clises $3^{2}$

For 4-connected objects there are 7 distinct of $\Im^{2}$ as shown in Figure 4

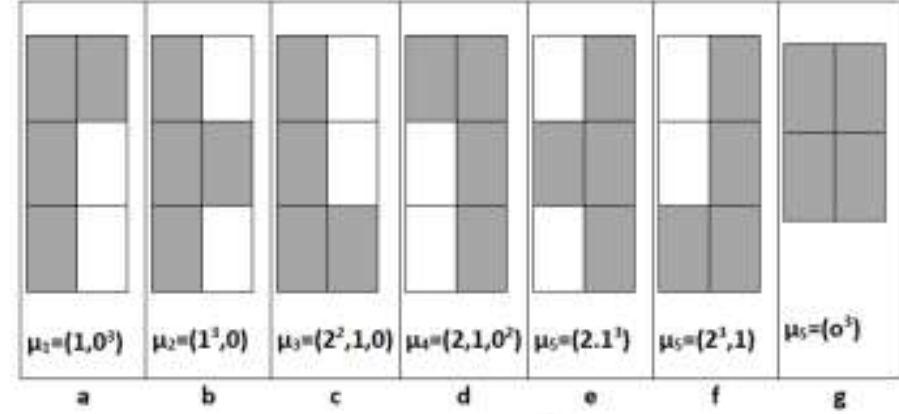

Figure 4: The 7 distinct shapes of $3^{2}$ for 4-oontuected sequares.

The theory and and concept in combinatorial to utilized to enumeration any $\Im^{2}$ for $n$-connected objects as given in the following theorem.

Theorem 3.4. Let $\Im^{2}$ be the nested chain abacus of $n$-connected objects with 2 columns and $r$ rows. Then, the are

$$
\begin{aligned}
& 2 \sum_{k=1}^{n-k}\left(\begin{array}{c}
n-k \\
k
\end{array}\right)+4\left(\sum_{d=0}^{k-2} \sum_{2 \leqslant k \leqslant n-k}\left(\begin{array}{c}
n-k-1 \\
d
\end{array}\right)+4\left[\sum_{i=2}^{n-2 k+2} \sum_{j=3}^{k} \sum_{k=3}\right.\right. \\
& \left(\left(\begin{array}{c}
n-2 k-i+1 \\
k-j
\end{array}\right)+k-3+2 \sum_{b=2}^{n-4} \sum_{a=1}^{k-3} \sum_{r=1}^{k-4} \sum_{k=5}^{n-1}\left[\begin{array}{c}
k=1 \\
a
\end{array}\right)+\left(\begin{array}{c}
n-k-b-2 \\
k-a-r-2
\end{array}\right)\right] \text { of } \Im^{2} \\
& \text { where } a, b, r, i \text { and } j \text { are positive integers for } 1 \leqslant k<n .
\end{aligned}
$$

Proof. Let $C_{1}$ and $C_{2}$ denote the number of bead positions in columns 1 and 2 respectively. Then, in $\Im^{2}$ we have $C_{1}=n-C_{2}$ such that $C_{2} \leqslant n-C_{2}$. The proof of this theorem is divided into three parts. In the first part we show how to get $\sum_{k=1}^{s-k}\left(\begin{array}{c}s-k \\ k\end{array}\right)$. There is a bijection from $C_{2}$ to $C_{1}$, so there are $\left(\begin{array}{c}C_{1} \\ C_{2}\end{array}\right)$ ways to connect $C_{2}$ with $C_{1}$. Meanwhile there are more than one bijections from $C_{1}$ to $C_{2}$ if $C_{2}=n-C_{1}$ and $C_{1} \leqslant n-C_{1}$. As $C_{2}$ varies, we obtain $\sum_{15 C_{15 C_{2}}}\left(\begin{array}{l}C_{1} \\ C_{2}\end{array}\right)$ ways. In general, if $k$ is the number of bead positions in one

column and $n-k$ is the number of bead positions in the other column, then there are $2 \sum_{\left.1 \leqslant k \leqslant n-k-1\left(n_{k}\right)^{*}\right)}$ of $\Im^{2}$.

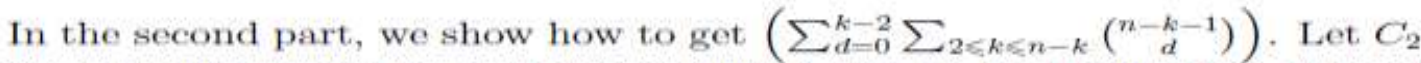
be the number of bead positions in column 2 and $C_{1}$ be the number of bead positions in column 1 where $C_{1}=n-C_{2}$ such that $C_{2} \leqslant C_{1}$. For this part we have two choices. The first choice is to observe that the bead positions are located in column 2 and are not connected with any bead positions in column 1. By definition of connectedness, $w_{2}$ is connected with $w_{1}$, hence there exist $\left(C_{1}-1\right)$ ways to connect $C_{1}$ bead positions with $C_{2}$ bead positions, where $d=C_{2}-2$. The second choice is to observe that $w_{n}$ is located in column 2 and not connected with any bead positions in $C_{1}$. By Remark ?? and Definition ?? $w_{b-2}$ is connected with $w_{b-3}$, thus there are $\left(C_{d}-1\right)$ ways to connect $C_{1}$ bead positions with $C_{2}$ bead positions, where $d=C_{2}-2$. As $d$ varies, we obtain $\sum_{d-0}^{C_{2}-2}\left(C_{1}-1\right)$ of $\Im^{2}$. On the other hand if $C_{2}=n-C_{1}$ and $C_{1} \leqslant C_{2}$ then there are $2 \sum_{d=0}^{C_{1}-2}\left(C_{2}-1\right)$ ways to connect $C_{1}$ bead positions with $\mathrm{C}_{2}$ bead positions. In general if $k$ is the number of bead positions in one column and $s-k$ is the number bead positions in the other column then there are $4\left(\sum_{2 \leqslant k s, n-k} \sum_{d=0}^{k-2}\left(\begin{array}{c}n-k \\ d\end{array}\right)\right)$ of $\Im^{2}$. In the final part of the proof, observe that the bead positions in $C_{1}$ and $C_{2}$ are not connected. There are $(\underset{k-j}{s-2 k-i+1})+k-3+$ 
$\left(\begin{array}{l}b \\ a\end{array}\right)+\left(\begin{array}{l}n-k-b-2 \\ k-a-r-2\end{array}\right)$ ways to connect $C_{1}$ with $C_{2}$. Since $k, i, j, r, a$ and $b$ vary then we obtain $4\left[\sum_{i=2}^{n-2 k+2} \sum_{j=3}^{k} \sum_{k=3}\left(\left(\begin{array}{c}n-2 k-i+1 \\ k-j\end{array}\right)+k-3\right)\right]+2 \sum_{b=2}^{s-k-4} \sum_{a=1}^{k-r-3}$ $\sum_{r=1}^{k-4} \sum_{k=5}^{n-k-1}\left[\left(\begin{array}{l}b \\ a\end{array}\right)+\left(\begin{array}{c}n-k-b-2 \\ k-a-r-2\end{array}\right)\right]$ ways to connect $C_{1}$ bead positions with $C_{2}$ bead positions. Hence, there exist a total of

$$
\begin{gathered}
2 \sum_{k=1}^{n-k}\left(\begin{array}{c}
n-k \\
k
\end{array}\right)+4\left(\sum_{d=0}^{k-2} \sum_{2 \leqslant k \leqslant n-k}\left(\begin{array}{c}
n-k-1 \\
d
\end{array}\right)+4\left[\sum_{i=2}^{n-2 k+2} \sum_{j=3}^{k} \sum_{k=3}\right.\right. \\
\left(\left(\begin{array}{c}
n-2 k-i+1 \\
k-j
\end{array}\right)+k-3+2 \sum_{b=2}^{n-k-4} \sum_{\substack{a-r-3 \\
\Im^{2}}}^{k-1} \sum_{r=1}^{k-4} \sum_{k=5}^{n-k-1}\left[\left(\begin{array}{l}
b \\
a
\end{array}\right)+\left(\begin{array}{c}
n-k-b-2 \\
k-a-r-2
\end{array}\right)\right]\right. \text { of } \\
\end{gathered}
$$

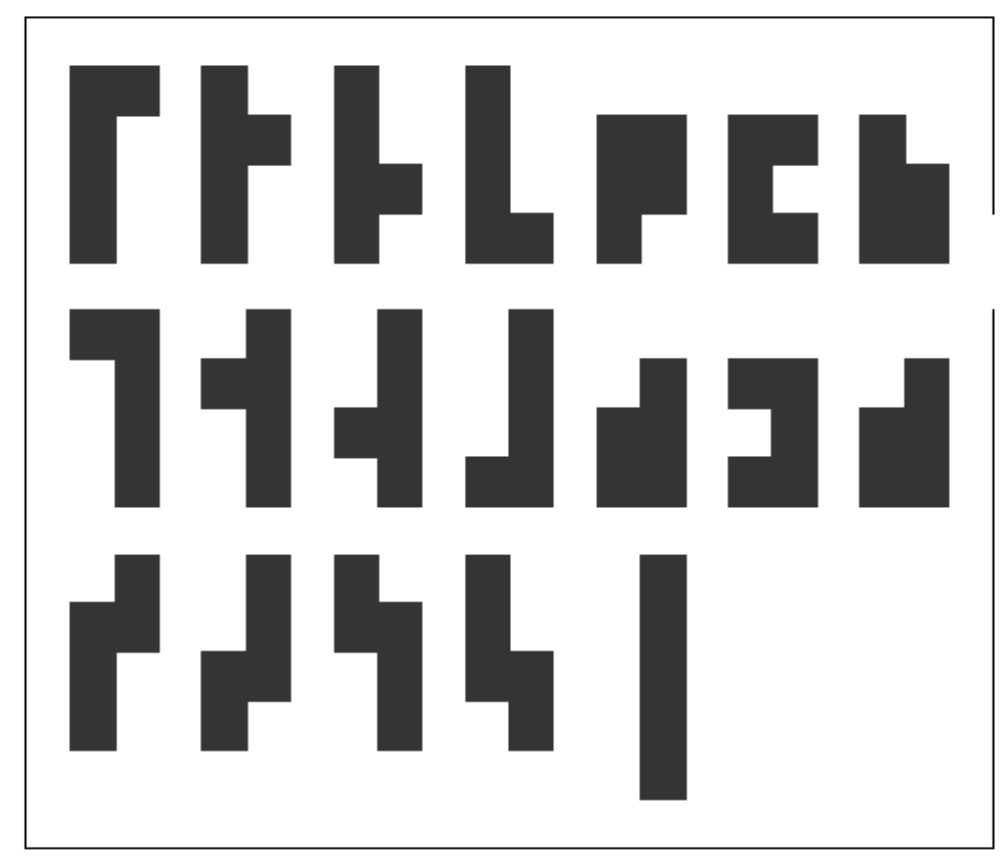

Figure 5: 5-connected beta numbers sequence with $e=2$

\section{Conclusion}

In this paper, it has been found that polyominoes is a spacial case of $e$-abacus. We introduced a new method to enumerate the $n$-connected squares by define new class .

\section{Reference}

[1] el Lungo, A., Duchi, E., Frosini, A., Rinaldi, S. (2003). Enumeration of convex polyominoes using the ECO method. In DMCS (pp. 103-116).

[2] oupil, A., Cloutier, H., Nouboud, F. (2010). Enumeration of polyominoes inscribed in a rectangle. Discrete Applied Mathematics, 158(18), 20142023.

[3] edelmeijer, D. H. (1981). Counting polyominoes: yet another attack. Discrete Mathematics, 36(3), 191-203.

[4] E. F. Mohommed, H. Ibrahem, N. Ahmad, A. Mahmood, Embedding chain movement in James abacus diagram for partitioning beta number, Proceedings of the 2nd Innovation and Analytics Conference Exhibition, 1691 (2015). 
[5] A. Mathas, Iwahori-Hecke algebras and Schur algebras of the symmetric group, American Mathematical Soc, 15(1999).

[6] D. Littlewood, Modular representations of symmetric groups. Proceedings of the Royal Society, A. Mathematical and Physical 41 Sciences, 209(1951), 333353.

[7] G. James, A. Kerber, The representation theory of the symmetric group, Reading, Mass (1981).

[8] D. G. James, Some combinatorial results involving young diagrams, Mathematical Proceedings of the Cambridge Philosophical Society, 83 (1978), 110 .

[9] Silva, T. O. E. 1999. Maximum excursion and stopping time recordholders for the $3 \mathrm{x}+1$ problem: computational results. Mathematics of Computation, 68(225), 371-384.

[10] Redelmeijer, D. H. 1981. Counting polyominoes: yet another attack. Discrete Mathematics, 36(3), 191-203.

[11] Gong, Kevin.2006 Polyominoes Enumeration. Kevin Gong. N.p., . Web. 24 Jul 2010, http://kevingong.com/Polyominoes/Enumeration.html.

[12] Jones, A. and Geagan, J. and Gong, K.L. and Periyannan, A. and Singer, D.W.1999. Method and apparatus for media data transmission. Google Patents. https://www.google.com/ patents/ WO1999037072A3?cl=en.

[13] Jones, Anne and Geagan, Jay and Gong, Kevin L and Periyannan, Alagu and Singer, David W. 2004. Method and apparatus for media data transmission. Google Patents.

[14] Dai, J. S., Zoppi, M., Kong, X. (2012). Advances in reconfigurable mechanisms and Robots I. Springer. 